in this technique of sound recording, in so far as a wider range of acoustic frequencies could be recorded with an absence of background noise such as hum and scratch. In the German magnetophone equip. ment, the recording medium is a plastic ribbon sprayed with a magnetic material in solution; this is much lighter and less bulky than the steel tape referred to above, and also has the advantage that imperfections can be removed and editing effected by cutting out the unwanted portions of the ribbon with scissors and sticking together again with a special fluid which sets almost immediately. The magnetic recording process would appear to be the same for the coated ribbon as for the steel tape, and while this magnetized ribbon can be kept indefinitely, it can as required be 'wiped clean' by passing it through a high-frequency magnetic field, after which the ribbon is ready for recording a new programme.

\section{Manufacture of Radio Equipment in India}

The August issue of the Journal of Scientific and Industrial Research published in Delhi contains an article by S. P. Chakravarti entitled "Manufacture of Wireless Apparatus in India". The growth of radio in India began in 1926, when two low-power broadcasting stations were set up in Bombay and Calcutta. In the following seven years radio telegraph and telephone beam stations were installed linking India with Great Britain and later with Japan. Since 1936, a broadcasting service has been operated on progressive lines under a separate department known as All India Radio. In spite of these developments, however, the production in India of radio equipment, including broadcasting receivers, prior to the outbreak of war in 1939, was confined to the assembly of sets from imported parts and components. In the following years these imports became increasingly difficult to obtain, and many firms had to abandon the manufacture of radio sets. In 1942, a systematic examination of the manufacturing position was carried out by the Government of India in connexion with the development of military radio sets. Investigations leading to the development of certain components from materials available in India have since been carried out under the auspices of the Radio Research Committee of the Board of Scientific and Industrial Research. It is now known that sufficient facilities for the manufacture of all components except valves exist in India, and that the organisations-some twenty in number-possessing manufacturing facilities are in urgent need of special basic materials and appliances.

The article referred to above gives details of these materials, and also a summary of the present manufacturing position of such components as carbon resistors, fixed and variable condensers, loudspeakers, transformers and vibrators. The need for the production of valves in India is stressed; and it is suggested that two factories, in the north and south respectively, should be immediately established for the production of valves with the aid of machinery imported from the United States or Britain. The number of radio receiving sets in India in 1944 was about 0.5 per 1,000 persons, and it is proposed that this should be increased to two sets per 1,000 persons during the next five to ten years by a production of about 100,000 sets a year. This is additional to the manufacture of large quantities of parts and replacements needed for maintaining and servicing about 200,000 broadcasting receivers and a number of communication receivers already in use in the country. In addition to the home consumption, the neighbouring countries-Burma, Malay States, Ceylon, China, Afghanistan and Persia-all of which have their own broadcasting stations, and also receive transmissions from India, may buy annually a total of 50,000 sets and a large number of components for their maintenance.

\section{A New Gem Stone}

A NEw mineral, brazilianite, which has possibilities as a gem stone, has just been announced jointly by Edward P. Henderson, of the Smithsonian Institution, and Frederick H. Pough, of the American Museum of Natural History. Obtained by Dr. Pough in Brazil from the owner, who thought it was chrysoberyl, the mineral on examination proved to have a different crystal habit from chrysoberyl, or from any other mineral. Brazilianite, which has been named after its country of origin, is a new monoclinic phosphate mineral, $\mathrm{Na}_{2} \mathrm{Al}_{6} \mathrm{P}_{4} \mathrm{O} \cdot{ }_{6}(\mathrm{OH})_{8}$. It is a yellowishgreen, glass-like mineral sufficiently good in colour and physical perfection to make it suitable for cutting into gem stones, although it is believed that it is not likely to become a popular gem because of its scarcity and lack of sufficient hardness to enable it to withstand much wear. Brazilianite is believed to be the first new mineral with gem-stone pnssibilities to be discovered since 1909, when benitoite was found in California. Messrs. Pough and Henderson have described brazilianite in a paper, with an illustration in colour, in the American Mineralogist (30, 572; 1945).

\section{Dutch Pharmacy under the German Occupation}

Pharmaceutical conditions in Holland under the German occupation were reviewed in a lecture given before the Pharmaceutical Society of Great Britain on December 13 by Dr. C. J. Blok, chief pharmacist to the University and municipal hospitals of Amsterdam. About 1943, said Dr. Blok, many articles became so scarce that the General Office of Public Health found it necessary to limit the prescribing of the doctors. Cod liver oil was only to be prescribed for tuberculosis ; atropine only for Parkin. sonism and eyedrops; pilocarpine only for eyedrops; luminal only for epilepsy ; pepsine for serious stomach cases ; dermatol only for epilepsy. After the Germans had robbed the safe of the Organon factories, insulin also became very scarce. Dr. Blok went into considerable detail regarding the means taken to economize alkaloids for eye treatment, including the use of ointments instead of solutions. In September 1944 came the loss of gas and electricity, which meant that sterilization in operating rooms by heat had to be reduced to a minimum as the spirit and petrol which might have been employed were very scarce. Fortunately he himself had steam autoclaves by which sterile injections could still be prepared. Operating rooms had to resort to chemical sterilization with 4 per cent 'Lysol', or a mixture of borax carbol-formalin with a little sodium nitrite against rusting; a very stable solution with quite effective sterilizing powers was 2 per cent formalin, $2 \cdot 5$ per cent phenol, 1.5 per cent borax. Another difficulty was the impossibility of melting ampoule glass, due to lack of gas. This was overcome by using an acetylene lamp made from an ordinary bottle; the temperature obtained was enough to melt glass, and the apparatus was used at home as a lamp. 